\title{
Evaluation of the primary medical treatments based on the advanced trauma life support principles in trauma patients
}

\author{
Payman Asadi ${ }^{1}$, Vahid Monsef Kasmaei ${ }^{1}$, Seyyed Mahdi Zia Ziabari², Siamak \\ Rimaz $^{3}$, Ehsan Modirian 4 , Ali Sarbazi-Golezari ${ }^{4,5}$ \\ ${ }^{1}$ Road Trauma Research Center, Guilan University of Medical Sciences, Rasht, Iran \\ ${ }^{2}$ Department of Emergency Medicine, School of Medicine, Guilan University of Medical \\ Sciences, Rasht, Iran \\ ${ }^{3}$ Department of Anesthesiology, Anesthesia Research Center, Alzahra Hospital, Guilan \\ University of Medical Sciences, Rasht, Iran \\ ${ }^{4}$ Department of Emergency Medicine, School of Medicine, Qazvin University of Medical \\ Sciences, Qazvin, Iran \\ ${ }^{5}$ Student Research Committee, Qazvin University of Medical Sciences, Qazvin, Iran \\ Corresponding Author: Siamak Rimaz, Department of Anesthesiology, Anesthesia Research \\ Center, Alzahra Hospital, Guilan University of Medical Sciences, Rasht, Iran. \\ Email:smkrimaz@yahoo.com
}

https://doi.org/10.1177/1460408620968337

\section{Abstract \\ Objective}

In Iran, road traffic accidents were responsible for 14716 deaths in 2015. This study aimed to compare the initial resuscitation of traumatically injured patients to the internationally recognized ATLS standards.

Materials and methods

As a cross-sectional study, 506 traumatically injured patients who were referred to the tertiary referral major trauma center in Poursina Hospital during the study period, were evaluated. All therapeutic interventions were compared to the ATLS standards. Data on mortality by demographic was compared to those in whom the ATLS standards were met and in those whom it was not met

Results

Mean age of the patients was $37.37 \pm 19.72$ and motorcycle was the most common cause of accidents (40.9\%). ATLS guideline interventions were completely performed in $18.2 \%$ of the patients in their primary hospital, and in rest of 414 cases $(81.8 \%)$, ATLS algorithms were not fully carried out. The mortality rate was significantly higher in the second group: $10.86 \%$ vs $32.36 \%$, respectively.

Conclusion Application of ATLS principles in multiple trauma patients can reduce the mortality rate.

\section{Keywords}

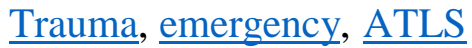

